\title{
Serologic Evidence of Zoonotic Alphaviruses in Humans from an Indigenous Community in the Peruvian Amazon
}

\author{
Jocelyn G. Pérez, ${ }^{1 \star}$ Jean-Paul Carrera, ${ }^{2}$ Emmanuel Serrano,,${ }^{3,4}$ Yaneth Pittí, ${ }^{2}$ Jorge L. Maguiña, ${ }^{5}$ Gregorio Mentaberre, ${ }^{3,6}$ \\ Andrés G. Lescano, ${ }^{5}$ Anayansi Valderrama, ${ }^{2}$ and Pedro Mayor ${ }^{3}$ \\ ${ }^{1}$ University of Liverpool, Liverpool, United Kingdom; ${ }^{2}$ Gorgas Memorial Institute of Health Studies, Panama City, Panama; ${ }^{3}$ Universitat Autònoma \\ de Barcelona, Barcelona, Spain; ${ }^{4}$ Universitá di Torino, Torino, Italy; ${ }^{5}$ Universidad Peruana Cayetano Heredia, Lima, Peru; ${ }^{6}$ Universitat de Lleida, \\ Lleida, Spain
}

\begin{abstract}
Alphaviruses (Togaviridae, Alphavirus) are arthropod-borne single-stranded RNA pathogens that cause febrile and neurologic disease in much of Latin America. However, many features of Alphavirus epidemiology remain unknown. In 2011, we undertook a cross-sectional study in Nueva Esperanza, an indigenous community in the Peruvian Amazon. Here, we present the first serologic evidence of Mayaro (MAYV), Venezuelan equine encephalitis (VEE) complex alphavirus, Una (UNAV), and Madariaga (MADV) viruses reported in humans (24\%, 16\%, 13\%, and 1.5\%, respectively) from an Amazonian indigenous community in Peru. Hunting activity and cohabiting with hunters were the main risk factors for Mayaro seroconversion, but only hunting was associated with UNAV seropositivity. Our results suggest that alphavirus infection in this region is common, but we highlight the high UNAV seroprevalence found and corroborate the low MADV prevalence reported in this region. Furthermore, MAYV-neutralizing antibodies were also detected in stored samples from wild animals (18\%) hunted by Nueva Esperanza inhabitants and another mestizo community located close to lquitos. Further serological surveys of VEE complex alphaviruses, UNAV, and MADV in wild animals and assessing the ability of the MAYV seropositive species to transmit the virus will be relevant.
\end{abstract}

\section{INTRODUCTION}

Arthropod-borne zoonotic alphaviruses (Togaviridae, Alphavirus) are positive-sense single-strand RNA viruses that are recognized as an important cause of acute febrile illness in humans in Latin America. In the Peruvian Amazon, Mayaro virus (MAYV) accounts for nearly $1 \%$ of outpatient evaluations for febrile disease, ${ }^{1}$ and cases have been reported in Latin American countries as well as in travelers returning from the Amazon Basin. ${ }^{2,3}$ Venezuelan equine encephalitis (VEE) complex alphaviruses, such as VEE virus (VEEV), have caused outbreak within the urban areas of lquitos, the largest city in the Peruvian Amazon, but few neurological cases and deaths have been observed. ${ }^{4,5}$ Less is known about Madariaga virus (MADV) infection in this region. Despite the enzootic circulation and intensive surveillance, only few seropositive individuals and no neurologic disease or fatal cases have been reported. ${ }^{6}$ However, encephalitis cases and mild or asymptomatic cases mostly affecting children have been attributed to MADV recently in Venezuela, Haiti, and Panama. ${ }^{7-10}$ Finally, although Una virus (UNAV) has been isolated from mosquitos of the Peruvian Amazon, it has been rarely associated with human disease. ${ }^{11,12}$ Therefore, its distribution and the human disease risk in this region remain unknown.

Although these alphaviruses are a well-recognized cause of human disease in the Americas, prior research studies were only carried out in the peri-urban area of lquitos. An important reason behind this is the challenge of conducting research in Amazonian communities because of different barriers (e.g., geographical, sociocultural, and health service access). ${ }^{13}$ The lack of surveillance studies in indigenous communities and the identification of potential wildlife hosts in the Amazon highlight the importance of

*Address correspondence to Jocelyn G. Pérez, University of Liverpool, 8 West Derby St., Liverpool L69 7BE, United Kingdom. E-mail: jgpl16@liverpool.ac.uk studying this region. Furthermore, the continuous ecological disturbance in the Amazon rainforest through human activities such as hunting, logging, and ecotourism may be significant risk factors for infection with these viruses. ${ }^{4,14}$ Therefore, we sought to investigate the epidemiology of these viruses through a cross-sectional serosurvey, involving humans and wildlife, in an indigenous community of the Peruvian Amazon.

\section{MATERIALS AND METHODS}

Human and animal survey. This study was conducted in Nueva Esperanza (S 04 ${ }^{\circ} 19.53$ W 71 ${ }^{\circ} 57.33$ ), 302 km northeast from lquitos (Figure 1). This is an indigenous community of 297 inhabitants, whose main occupations are small-scale agriculture, fishing, logging, and subsistence hunting. In 2011, we collected human serum samples from 70 volunteers (ranged from age 5 to 81 years) of this community. Between 2007 and 2008, we also collected blood samples from 90 wild animals harvested by local hunters from Nueva Esperanza and Sol Naciente (S $03^{\circ} 38.26$ W $73^{\circ} 12.57$ ), a mestizo community located $26 \mathrm{~km}$ from lquitos where agricultural land use has partially replaced the forest.

Laboratory testing. Human samples were first tested by plaque reduction neutralization tests (PRNT) against MAYV (strain TRVL15537), MADV (chimeric SIN/MADV derived from Brazilian MADV strain BeAn436087), and VEE complex alphavirus (vaccine strain TC-83). ${ }^{15,16}$ All samples with PRNT80 titers $\geq 20$ were considered positive. To complement PRNT results and as a secondary goal because of the low serum volume available, the leftover sample was tested in triplicate for lgG antibodies to antigens of MAYV, MADV, and VEE complex viruses. We used a capture ELISA. ${ }^{17}$ For the ELISA, the TRVL15537 strain was used for MAYV and sucrose-acetone antigens were prepared from MADV- (prepared by Dr. Robert Shope at the Yale Arbovirus Research Unit in August 1989), and VEE complex alphaviruses (vaccine strain TC-83 and Mosso das Pedras 78V3531) infected mouse 


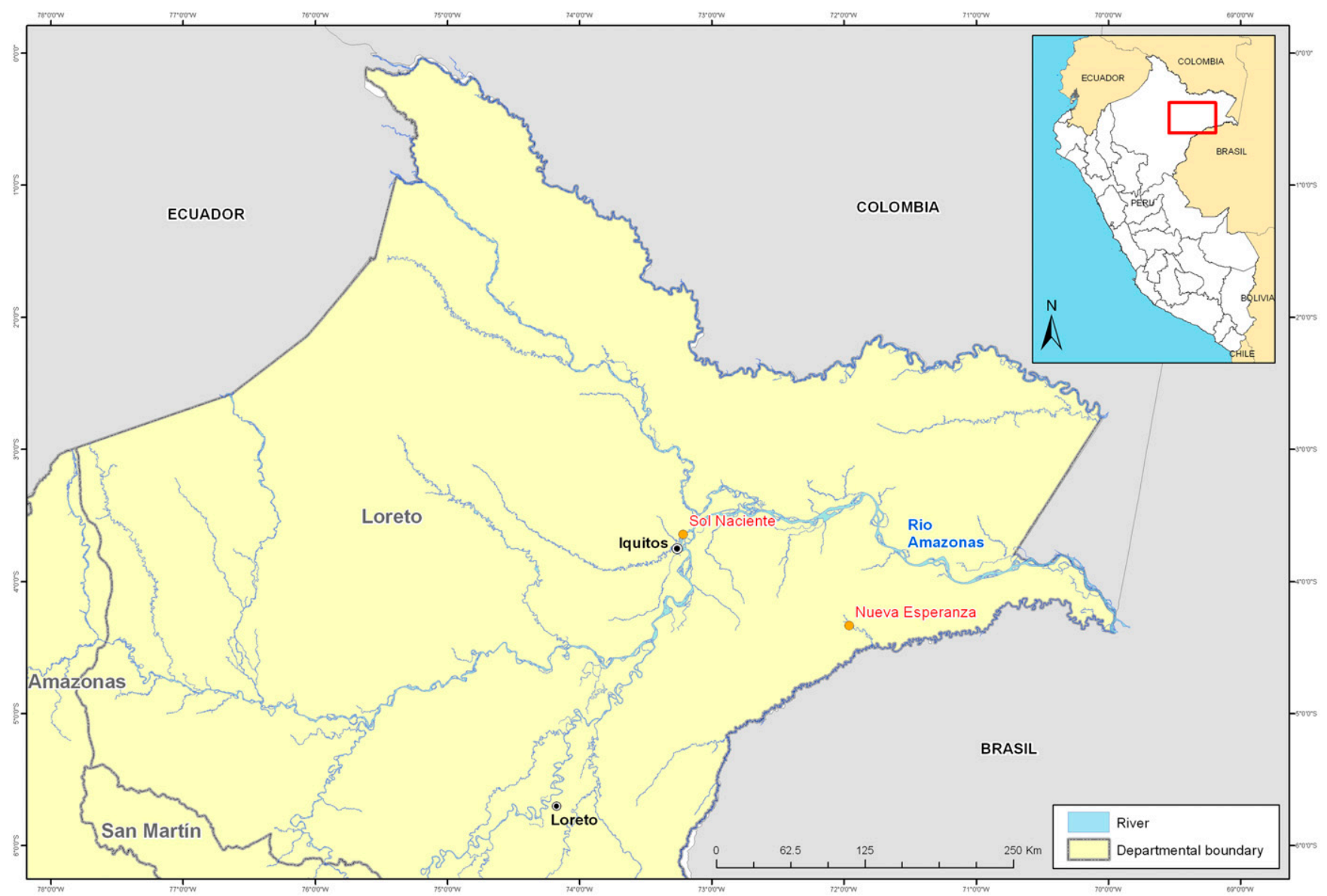

FIGURE 1. Nueva Esperanza and Sol Naciente communities in the Department of Loreto, Peru. This figure appears in color at www.ajtmh.org.

brain (provided by the US CDC and University of Texas Medical Branch (UTMB) World Reference Center for Emerging Viruses and Arboviruses, respectively). To discriminate MAYV positives from cross-reactivity with UNAV, the leftover from all the samples was first screened in 1:20 dilution of the UNAV strain BT-1495-3 and then tested by PRNT80. Chikungunya virus was not tested because these samples were collected in 2011, before chikungunya virus circulated in the Americas.

Wild animal samples were tested only by PRNT against MAYV (strain TRVL15537). Plaque reduction neutralization tests for VEE complex alphaviruses, MADV, and UNAV could not be performed because of insufficient samples.

Statistical analysis. We used a recursive modeling approach with classification trees to identify major risk factors linked to the presence of antibodies against these alphaviruses using the package "rpart" version 4.1-13 of the statistical software $R$ version 3.4.2. ${ }^{18,19}$ Risk factors evaluated for prior human infection were gender, hunting activity, logging activity, and cohabitation with seropositive people. Model simplification takes into consideration the complexity parameter of the tree showing the minimum relative error after 10-fold cross-validations.

Ethics. Research protocols were approved by the Peruvian Forestry and Wildlife Agency (041-2007-DGFFS), the Institutional Animal Care and Use Committee of U.S. Naval Medical Research Unit No. 6, and Instituto Nacional de Salud del Perú (\#08-982).

\section{RESULTS}

Characteristics of the human population. A total of 70 participants (23.6\% of local inhabitants) who belong to 22 houses (48.9\% of total houses) were surveyed. The age range was 5-81 years, with a median age of $20\left(Q_{1}=10\right.$ and $Q_{3}=$ 34.5). The proportion of females was $58.8 \%$. A total of $18.6 \%$ of participants declared to hunt, whereas $14.3 \%$ declared to participate in logging activities. The total number of persons per house ranged between one and seven (Table 1).

Human seroprevalence. Based on our PRNT results, the seroprevalence for MAYV, VEE complex alphavirus, UNAV, and MADV in people from Nueva Esperanza was 24\% $(17 / 70$, 95\% Cl [16-35]), 16\% (11/67, 95\% Cl [9-27]), 13\% (6/48, 95\% $\mathrm{Cl}$ [6-25]), and $1.5 \%(1 / 67,95 \% \mathrm{Cl}$ [0-8]), respectively (Supplemental Table 1). For MAYV, the most parsimonious tree model explained $27 \%$ of likelihood of showing antibodies against MAYV, including two risk factors: hunting activity and cohabitation with seropositive people (Figure 2). This means that being a hunter $(75 \%, 95 \% \mathrm{Cl}$ [42.81-94.51]) or not being a hunter but sharing home with MAYV seropositive people $(21 \%, 95 \% \mathrm{Cl}$ [9.5-37.3]) were the main risk factor associated with MAYV circulation. Individuals who were nonhunters and not living in a seropositive household were seronegative. The UNAV tree model also showed hunting activity as the main risk factor $(80 \%, 95 \% \mathrm{Cl}$ [28.4-99.5]) for the virus infection, explaining $49 \%$ of the observed UNAV seropositive variability. 
TABLE 1

Characteristics of PRNT- positive individuals $(n=40)$ and laboratory findings*

\begin{tabular}{|c|c|c|c|c|c|c|c|c|c|c|c|c|c|c|c|c|c|}
\hline \multirow[b]{2}{*}{ Sample ID } & \multirow[b]{2}{*}{ Gender } & \multirow[b]{2}{*}{ Age (years) } & \multirow[b]{2}{*}{ Hunter } & \multirow[b]{2}{*}{ Logger } & \multirow[b]{2}{*}{ House } & \multicolumn{3}{|c|}{ Mayaro virus } & \multicolumn{3}{|c|}{ Madariaga virus } & \multicolumn{3}{|c|}{$\begin{array}{c}\text { Venezuelan equine } \\
\text { encephalitis complex virus }\end{array}$} & \multicolumn{3}{|c|}{ Una virus } \\
\hline & & & & & & PRNT & PRNT titer & ELISA & PRNT & PRNT titer & ELISA & PRNT & PRNT titer & ELISA & Screen PRNT & PRNT & PRNT titer \\
\hline MIS0963 & $\mathrm{F}$ & 21 & No & No & A & - & - & - & - & - & + & + & 20 & + & - & - & - \\
\hline MIS0965 & $\mathrm{F}$ & 35 & No & No & A & + & 20 & + & NP & NP & NP & NP & NP & NP & NP & NP & NP \\
\hline MIS0967 & M & 41 & Yes & Yes & A & - & - & + & - & - & + & - & - & - & + & + & 20 \\
\hline MIS0968 & $\mathrm{F}$ & 33 & No & No & A & + & 40 & + & - & - & NP & - & - & NP & NP & NP & NP \\
\hline MIS0969 & $M$ & 8 & No & No & A & + & 40 & + & - & - & NP & - & - & NP & NP & NP & NP \\
\hline MIS0972 & M & 7 & No & No & D & - & - & + & - & - & + & + & 80 & - & - & - & - \\
\hline MIS0974 & M & 67 & Yes & No & E & - & - & + & - & - & - & - & - & - & + & + & 80 \\
\hline MIS0975 & $\mathrm{F}$ & 64 & No & No & E & + & 160 & + & - & - & + & - & - & - & NP & NP & NP \\
\hline MIS0977 & M & 29 & No & Yes & $\mathrm{F}$ & - & - & + & - & - & NP & + & 80 & NP & NP & NP & NP \\
\hline MIS0980 & M & 81 & Yes & No & $\mathrm{F}$ & + & 160 & + & - & - & - & - & - & - & + & + & 80 \\
\hline MIS0983 & M & 70 & Yes & No & $\mathrm{G}$ & + & 40 & + & - & - & -- & - & - & - & + & + & 40 \\
\hline MIS0985 & M & 33 & No & Yes & $\mathrm{H}$ & + & 20 & + & - & - & + & - & - & - & NP & NP & NP \\
\hline MIS0986 & M & 30 & Yes & No & $\mathrm{H}$ & + & 40 & + & NP & NP & NP & NP & NP & NP & NP & NP & NP \\
\hline MIS0988 & M & 39 & Yes & Yes & I & + & 20 & + & - & - & NP & - & - & NP & NP & NP & NP \\
\hline MIS0990 & $\mathrm{F}$ & 32 & No & No & $\mathrm{J}$ & - & - & - & - & - & - & - & - & - & + & + & 40 \\
\hline MIS0991 & $\mathrm{F}$ & 26 & No & No & $\mathrm{K}$ & - & - & + & - & - & + & + & 120 & - & NP & NP & NP \\
\hline MIS0992 & $\mathrm{M}$ & 10 & No & No & $\mathrm{K}$ & + & 40 & + & - & - & NP & - & - & NP & NP & NP & NP \\
\hline MIS0994 & $\mathrm{F}$ & 20 & No & No & L & - & - & + & - & - & - & - & - & - & - & - & - \\
\hline MIS0995 & M & 45 & Yes & Yes & L & + & 40 & + & + & 40 & NP & - & - & NP & NP & NP & NP \\
\hline MIS1000 & $\mathrm{F}$ & 31 & No & No & M & - & - & + & - & - & + & + & 40 & + & + & + & 80 \\
\hline MIS1001 & $\mathrm{F}$ & 6 & No & No & $M$ & - & - & + & - & - & - & - & - & - & - & - & - \\
\hline MIS1004 & $\mathrm{F}$ & 25 & No & No & $\mathrm{N}$ & - & - & + & - & - & - & - & - & - & - & - & - \\
\hline MIS1006 & M & 52 & Yes & Yes & $\mathrm{N}$ & + & 40 & + & - & - & NP & - & - & NP & NP & NP & NP \\
\hline MIS1007 & M & 46 & Yes & Yes & $\mathrm{N}$ & + & 20 & + & - & - & NP & - & - & NP & NP & NP & NP \\
\hline MIS1008 & $\mathrm{F}$ & 13 & No & No & $\mathrm{O}$ & - & - & + & - & - & - & + & 160 & + & - & - & - \\
\hline MIS1011 & M & 30 & Yes & Yes & Q & - & - & + & - & - & + & - & - & - & NP & NP & NP \\
\hline MIS1013 & M & 13 & No & No & $\mathrm{R}$ & - & - & + & - & - & - & - & - & - & - & - & - \\
\hline MIS1014 & $\mathrm{M}$ & 10 & No & No & S & - & - & + & - & - & + & + & 40 & + & - & - & - \\
\hline MIS1018 & $\mathrm{F}$ & 7 & No & No & $\mathrm{T}$ & - & - & + & - & - & - & - & - & - & - & - & - \\
\hline MIS1021 & M & 18 & Yes & No & $U$ & - & - & + & NP & NP & NP & NP & NP & NP & NP & NP & NP \\
\hline MIS1022 & M & 47 & No & Yes & $U$ & + & 20 & + & - & - & NP & - & - & NP & NP & NP & NP \\
\hline MIS1023 & M & 74 & Yes & No & $U$ & + & 20 & + & - & - & NP & - & - & NP & NP & NP & NP \\
\hline MIS1024 & M & 67 & Yes & No & Z & + & 40 & + & - & - & - & + & 40 & + & - & - & - \\
\hline MIS1025 & $\mathrm{F}$ & 8 & No & No & Z & - & - & + & - & - & + & + & 80 & + & - & - & - \\
\hline MIS1026 & $\mathrm{F}$ & 20 & No & No & Z & - & - & + & - & - & + & + & 80 & + & NP & NP & NP \\
\hline MIS1028 & M & 20 & No & No & Z & - & - & + & - & - & + & - & - & - & - & - & - \\
\hline MIS1029 & $\mathrm{F}$ & 17 & No & No & NA & + & 20 & + & - & - & NP & - & - & NP & NP & NP & NP \\
\hline MIS1030 & $\mathrm{F}$ & 6 & No & No & NA & - & - & + & - & - & - & - & - & - & - & - & - \\
\hline MIS1031 & $\mathrm{F}$ & 12 & No & No & NA & - & - & + & - & - & - & - & - & - & - & - & - \\
\hline MIS1032 & $\mathrm{F}$ & 35 & No & No & NA & - & - & + & - & - & + & + & 40 & - & NP & NP & NP \\
\hline
\end{tabular}

$\mathrm{M}=$ males; $\mathrm{F}$ = females; $\mathrm{NA}$ = data not available; $\mathrm{NP}$ = not performed because of insufficient sample; $\mathrm{PRNT}$ = plaque reduction neutralization test.

People who did not report hunting had a lower UNAV seroprevalence of $4-7 \%(95 \% \mathrm{Cl}$ [0.6-16.2]). No other variables were associated. Una virus seropositives were also exposed to MAYV or other alphavirus, but some individuals only showed exposure to UNAV. Despite the high seroprevalence (16\%) of VEE complex virus, the tree models showed that none of the studied variables were related to the positive cases. Risk factors to MADV infection were not evaluated because of concerns about the representativeness of the sample because only one sample was found seropositive.

Animal seroprevalence. Anti-MAYV-neutralizing antibodies were detected in wild animals (18\%, 95\% Cl [11-27\%]) collected near Nueva Esperanza and Sol Naciente communities. The highest MAYV seroprevalence in wild animals was observed in armadillos (40\%), followed by nonhuman primates (33\%), peccaries (11\%), and rodents (10\%) (Table 2).

\section{DISCUSSION}

Recent epidemiological and surveillance studies have shown that human alphavirus infections in Latin America are common. ${ }^{2,16,20}$ Herein, we demonstrate endemic infection with new world alphaviruses in the Amazon region of Peru. In addition, we provide serologic results of potential wildlife hosts of MAYV.

Madariaga virus is the new designation for the South American isolates of eastern equine encephalitis virus. ${ }^{21}$ In South America, this virus has caused equine encephalitis outbreaks, but only two human cases were detected to date (in Brazil and Trinidad). ${ }^{6}$ The low MADV seroprevalence observed in this study corroborates a previous study in Iquitos that showed a seroprevalence of $3 \%(9 / 359) .{ }^{6}$ This result is in contrast to the high human seroprevalence and cases reported in Panama. ${ }^{8}$ Cross-protective antibodies may be one of the factors limiting the infection and disease in South America. ${ }^{6}$ Although the virus was isolated from Culex mosquitoes in areas near lquitos, our study found only one seropositive individual, a 45-year-old male hunter and logger who also showed exposure to MAYV. ${ }^{11}$ Evidence of exposure to only MADV is unusual: most seropositive individuals also show exposure to MAYV, VEEV, or UNAV. ${ }^{6}$ 
A Hunter

$\begin{array}{cc}\text { No } & \text { Yes } \\ \text { Cohabit } & \begin{array}{c}0.69 \\ n=13\end{array}\end{array}$

0
$n=8$ Logging

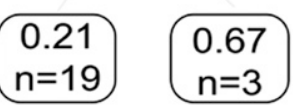

B

Bunter

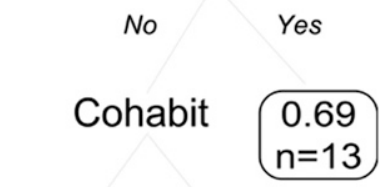

0
$n=8$$\quad \begin{gathered}0.27 \\ n=22\end{gathered}$

FIGURE 2. Complete (A) and final (B) classification trees exploring the influence of gender and hunting or logging behaviors on the probability of showing antibodies against Mayaro virus in the Peruvian Amazon forest. The factor "cohabit" indicates people who share home with a Mayaro seropositive person. Note that gender was initially retained but later excluded after model simplification. Left branches indicate no hunters or no cohabitation, whereas the right branches summarize the probability for male hunters or cohabiting people.

Venezuelan equine encephalitis complex viruses such as VEEV are known to cause human disease in the Peruvian Amazon. The most recent VEEV outbreak caused by the enzootic Panama/Peru ID genotype in lquitos, in early 2006, accounted for 63 cases and one fatality. ${ }^{4,5}$ In contrast, a higher fatality rate was reported in Panama (5\%, two deaths from 39 cases studied). Different factors may be associated with this difference: a more active surveillance in Panama during outbreaks, underreporting of VEEV cases in Peru, or other factors involved in the emergence of the enzootic VEEV (virus adaptation to mosquito species or mosquito abundance). ${ }^{22}$ In this study, the vaccine strain TC-83, closely related to subtype ID, was used for the PRNT. Unfortunately, PRNTs were not performed using other VEE complex viruses that are geographically relevant, limiting our ability to definitively state which VEE complex virus was involved in these cases. Interestingly, the VEE complex virus titers are low, presumably because of the infection with sympatric alphaviruses inducing PRNT cross-reaction. However, antibody titers waning over time is also a possibility, as most of the VEE complex virus positives are in older age.

Mayaro infections have been principally recognized during outbreaks in South America; however, risk factors for the infection and the clinical spectrum of MAYV infections are poorly understood. ${ }^{1,23-26}$ The high seroprevalence (24\%) found in our study corroborates previous studies in indigenous groups of the Brazilian Amazon, where MAYV seroprevalence ranged from $20 \%$ to $47 \%$, with an overall of $42 \%$ for all the groups together. ${ }^{27} \mathrm{~A}$ greater seroprevalence was also found in ethnic groups of French Guiana living in contact with the forest compared with those who do not. ${ }^{28}$ Despite the growing concern of MAYV as an emerging virus that may potentially spread and cause human outbreaks in the Western Hemisphere, less is known about the virus ecology. ${ }^{29}$ Most evidence comes from Brazilian studies, which proposed

TABLE 2

Mayaro virus seroprevalence in wild animals hunted by Nueva Esperanza and Sol Naciente communities in the northeastern Peruvian Amazon

\begin{tabular}{|c|c|c|c|c|}
\hline & & Common name & Scientific name & No. PRNT positive $(\%)^{\star}$ \\
\hline \multirow[t]{22}{*}{ Mammals } & Edentate & Nine-banded armadillo & Dasypus novemcinctus & 2/4 (50.0) \\
\hline & & Three-toed sloth & Bradypus sp. & $0 / 1(0.0)$ \\
\hline & & & Subtotal & $2 / 5(40)$ \\
\hline & Primate & Poeppig's woolly monkey & Lagothrix poeppigii & $6 / 11(54.5)$ \\
\hline & & Red howler monkey & Alouatta seniculus & $1 / 1(100.0)$ \\
\hline & & Red uakari & Cacajao calvus & $1 / 3(33.3)$ \\
\hline & & Large-headed capuchin & Sapajus macrocephalus & $1 / 6(16.7)$ \\
\hline & & Squirrel monkey & Saimiri macrodon & $0 / 3(0.0)$ \\
\hline & & White-fronted capuchin & Cebus albifrons & $0 / 2(0.0)$ \\
\hline & & Monk saki & Pithecia monachus & $0 / 1(0.0)$ \\
\hline & & & Subtotal & $9 / 27(33)$ \\
\hline & Artiodactyla & Collared peccary & Pecari tajacu & $1 / 6(16.7)$ \\
\hline & & Red brocket deer & Mazama americana & $0 / 3(0.0)$ \\
\hline & & & Subtotal & $1 / 9(11)$ \\
\hline & Rodent & Wild black agouti & Dasyprocta fuliginosa & 3/27 (11.1) \\
\hline & & Lowland paca & Cuniculus paca & 1/10 (10.0) \\
\hline & & Northern Amazon red squirrel & Sciurus igniventris & $0 / 1(0.0)$ \\
\hline & & Spiny rat & Proechimys sp. & $0 / 1(0.0)$ \\
\hline & & & Subtotal & $4 / 39(10)$ \\
\hline & Carnivora & South American coati & Nasua nasua & $0 / 4(0.0)$ \\
\hline & & & Subtotal & $0 / 4(0)$ \\
\hline & Subtotal & & & 16/84 (19) \\
\hline \multirow[t]{5}{*}{ Birds } & & Blue-and-yellow macaw & Ara ararauna & $0 / 2(0.0)$ \\
\hline & & Great tinamou & Tinamus major & $0 / 2(0.0)$ \\
\hline & & Rufescent tiger heron & Tigrisoma lineatum & $0 / 1(0.0)$ \\
\hline & & Speckled chachalaca & Ortalis guttata & $0 / 1(0.0)$ \\
\hline & Subtotal & & & $0 / 6(0)$ \\
\hline Total & & & & 16/90 (18) \\
\hline
\end{tabular}

PRNT = plaque reduction neutralization test.

* Samples were first tested with an ELISA for the presence of Mayaro virus antibodies, and in those screening positive, a confirmatory PRNT was performed. 
nonhuman primates as the most important amplifying hosts. ${ }^{23}$ The virus was isolated from a marmoset (Callithrix sp.) captured during an MAYV outbreak in Brazil, and experimental infections in these animals confirmed the occurrence of viremia post-MAYV infection. ${ }^{23}$ The virus was also isolated from only one migrating bird (Icterus spurius) from a total of 1,300 birds studied in the United States in $1967 . .^{30}$ Serologic studies found MAYV antibodies in other sylvatic vertebrates and birds, but their exact role in the transmission of the virus is not determined. $^{31-33}$ This study detected anti-MAYV-neutralizing antibodies in wild animal species, which are consistent with previously described epidemiologic patterns of the sylvatic cycle, where arboreal and diurnal wild animals were more likely to be infected. ${ }^{32}$ Nevertheless, other terrestrial and nocturnal species were also seropositive in our study. Similar to our findings, previous studies indicated that nonhuman primates had high MAYV seropositive rates, presumably reflecting these animals' preference for a lower to mid-canopy habitat, where potential vectors are often present. ${ }^{23,32} \mathrm{We}$ also found seropositive rodents and marsupials, possibly infected by Haemagogus janthinomys mosquitoes, known enzootic vector of MAYV that reside in upper and lower canopy in the Peruvian Amazon. ${ }^{34}$ These mosquitoes are daytime feeders, which may explain why hunting was a risk factor. This result corroborates a previous study in the Amazon rainforest of Ecuador, where the high seroprevalence detected in the Amazon-native personnel of the Ecuadorian armed forces was associated with hunting. ${ }^{14}$ The higher MAYV risk due to cohabitation may suggest the presence of infected dwelling species, opening up the possibility for a human-mosquitohuman transmission cycle. Although $H$. janthinomys is reported to have a low activity near houses in the Amazon rainforest, the lack of MAYV isolation from other mosquito species and vector competence studies are unable to confirm a human-mosquito-human transmission. ${ }^{23,35}$ Mayaro virus has been isolated from Aedes (Ochlerotatus) serratus, Psorophora ferox, Psorophora albipes, and Culex spp. captured indoor and outdoor in a village in the lquitos region and at the forest area. ${ }^{36}$ Evaluating the capacity of these species to transmit the virus is highly relevant. Urban-adapted species such as Aedes aegypti and Aedes albopictus, known vectors of other human arboviruses (e.g., dengue and chikungunya) in lquitos, are proved competent MAYV vectors in laboratory conditions. However, other species, for example, Anopheles spp., may also be important vectors that sustain the transmission of MAYV. ${ }^{35,37}$

Here, we also demonstrate the highest observed human prevalence of UNAV reported to date in Latin America. Low UNAV prevalence was previously reported in lquitos and periurban and urban areas in Argentina and Paraguay where it is

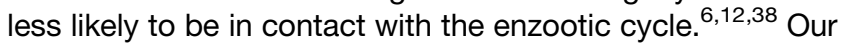
results show that hunting was a main risk factor for UNAV infection, and this suggests that introduction into the forest potentially increases the contact with humans and the transmission cycle. Ecological evidence suggests that $\mathrm{Ae}$. (Ochlerotatus) fulvus, P. ferox, and Psorophora albigenu might be the mosquito species involved in the enzootic circulation of UNAV, which is principally distributed in sylvatic areas of Latin American countries. ${ }^{11}$

Although disease severity varies between individuals, the chronic debilitating joint pain associated with Mayaro fever may severely impair the livelihoods of MAYV-infected people, whereas VEEV may cause fatal encephalitis. ${ }^{1}$ Unfortunately, no medical history was available to associate the seropositivity with the presence of chronic arthralgia or neurologic disease.

Although wild animals may have contributed to the amplification of MAYV during past Amazonian outbreaks, reservoir hosts for MADV, UNAV, and VEE complex alphaviruses in the Peruvian Amazon remain unknown. ${ }^{23}$ We also do not rule out the possibility that some MAYV seropositive animal samples could be due to a cross-reaction with UNAV; however, this hypothesis could not be tested because of insufficient samples. In humans, we found antibodies against both MAYV and UNAV in two individuals, but most MAYV seropositives were not tested for UNAV. The sample limitation did not allow us to indicate a reliable proportion of MAYV/ UNAV cross-reactions. It is unclear whether the crossneutralizing patterns shown in these two individuals are due to cross-reaction among these viruses or real independent infections. Future studies should evaluate this possibility in a prospective follow-up of patients.

The cross-sectional nature of the study design does not allow us to draw a conclusion of temporal association between the exposure to the virus and infection. However, our study was designed to explore potential factors of alphavirus infection and not to test hypothesis-driven questions. As sympatric alphaviruses circulate in the Amazon region of Peru, it is possible to observe cross-reaction among members of the genus Alphavirus. ${ }^{8,39}$ However, we used PRNT, which is the gold standard for alphavirus diagnosis and provides a highly accurate classification among members of the same antigenic complex.

In summary, we provide the first evidence of human alphavirus infection in an indigenous community from the Peruvian Amazon. Our results also suggest that sympatric human alphavirus infections are common in the Amazon region of Peru.

Received October 23, 2018. Accepted for publication August 15, 2019.

Published online September 30, 2019.

Note: Supplemental table appears at www.ajtmh.org.

Acknowledgments: We thank the citizens of Nueva Esperanza and Sol Naciente. We are especially grateful for the institutional support provided by Wildlife Conservation Society, Servicio Nacional de Sanidad Agraria, Instituto Nacional de Salud, and Dirección General Forestal y de Fauna Silvestre from Peru. We also thank Eric Halsey, Carolina Guevara, and Julia Ampuero for their support in the execution of this study and Karen Neira and Stephanie Montero for their technical assistance.

Financial support: This research was supported by a grant from SENACYT FID 16-201 to J. P. C. and A. V., Secretaría Nacional de Ciencia y Tecnología from Panama; by the grant for neglected diseases studies in Panama 1.11.1.3.703.01.55.120 from the Ministry of Economy and Finance of Panama to J. P. C. and A. V.; and by ERANet17/HLH-0271. Scott Weaver also supported this study through the World Reference Center for Emerging Viruses and Arboviruses, NIH grant R24Al120942. Dr. Lescano is sponsored by the training grant D43 TW007393 awarded by the Fogarty International Center of the US National Institutes of Health. J. L. M. is a doctoral candidate studying an Epidemiological Research Doctorate at Universidad Peruana Cayetano Heredia under FONDECYT/CIENCIACTIVA scholarship EF033-235-2015 and also supported by training grant D43 TW007393. A. V. is a member of the Sistema Nacional de Investigación de Panamá (SNI), SENACYT. E. S. was supported by the Spanish Ministerio de Ciencia Innovación y Universidades (MICINN) through a Ramon y Cajal agreement (RYC-2016-21120). 
Authors' addresses: Jocelyn G. Pérez, University of Liverpool, Liverpool, United Kingdom, E-mail: jgpl16@liverpool.ac.uk. Jean-Paul Carrera, Yaneth Pittí, and Anayansi Valderrama, Gorgas Memorial Institute of Health Studies, Panama City, Panama, E-mails: jpcarrera@ gorgas.gob.pa, ypitti@gorgas.gob.pa, and avalderrama@gorgas. gob.pa. Emmanuel Serrano, Universitat Autònoma de Barcelona, Barcelona, Spain and Universitá di Torino, Torino, Italy, E-mail: emmanuel.serrano@uab.cat. Jorge L. Maguiña and Andres G. Lescano, Universidad Peruana Cayetano Heredia, Lima, Peru, E-mails: jorge.luis.maguina@upch.pe and willy.lescano@upch.pe. Gregorio Mentaberre, Universitat Autònoma de Barcelona, Barcelona, Spain and Universitat de Lleida, Lleida, Spain, E-mail: gregorio.mentaberre@uab. cat. Pedro Mayor, Universitat Autònoma de Barcelona, Barcelona, Spain, E-mail: mayorpedro@hotmail.com.

\section{REFERENCES}

1. Halsey ES, Siles C, Guevara C, Vilcarromero S, Jhonston EJ, Ramal C, Aguilar PV, Ampuero JS, 2013. Mayaro virus infection, Amazon basin region, Peru, 2010-2013. Emerg Infect Dis 19: $1839-1842$.

2. Forshey BM et al., 2010. Arboviral etiologies of acute febrile illnesses in western South America, 2000-2007. PLoS Negl Trop Dis 4: e787.

3. Neumayr A, Gabriel M, Fritz J, Gunther S, Hatz C, SchmidtChanasit J, Blum J, 2012. Mayaro virus infection in traveler returning from Amazon basin, northern Peru. Emerg Infect Dis 18: 695-696.

4. Morrison AC et al., 2008. Venezuelan equine encephalitis virus in Iquitos, Peru: urban transmission of a sylvatic strain. PLoS Negl Trop Dis 2: e349.

5. Vilcarromero $S$ et al., 2010. Venezuelan equine encephalitis and 2 human deaths, Peru. Emerg Infect Dis 16: 553-556.

6. Aguilar PV et al., 2007. Endemic eastern equine encephalitis in the Amazon region of Peru. Am J Trop Med Hyg 76: 293-298.

7. Luciani K, Abadía I, Martínez-Torres AO, Cisneros J, Guerra I, García M, Estripeaut D, Carrera JP, 2015. Case report: Madariaga virus infection associated with a case of acute disseminated encephalomyelitis. Am J Trop Med Hyg 92: 1130-1132.

8. Carrera JP et al., 2013. Eastern equine encephalitis in Latin America. N Engl J Med 369: 732-744.

9. Lednicky JA, White SK, Mavian CN, El Badry MA, Telisma T, Salemi M, Okech BA, Beau De Rochars VM, Morris JG Jr., 2019. Emergence of Madariaga virus as a cause of acute febrile illness in children, Haiti, 2015-2016. PLoS Negl Trop Dis 13: e0006972.

10. Blohm GM, Lednicky JA, White SK, Mavian CN, Márquez MC, González-García KP, Salemi M, Morris JG Jr., Paniz-Mondolfi $A E, 2018$. Madariaga virus: identification of a lineage III strain in a Venezuelan child with acute undifferentiated febrile illness, in the setting of a possible equine epizootic. Clin Infect Dis 67: 619-621.

11. Turell MJ et al., 2005. Isolation of viruses from mosquitoes (Diptera: Culicidae) collected in the Amazon basin region of Peru. $J$ Med Entomol 42: 891-898.

12. Diaz LA, Spinsanti LI, Almiron WR, Contigiani MS, 2003. UNA virus: first report of human infection in Argentina. Rev Inst Med Trop Sao Paulo 45: 109-110.

13. Palma-Pinedo H, Reyes-Vega MF, 2018. Barriers identified by the health staff for the screening of human immunodeficiency virus in indigenous populations of the peruvian amazon [article in Spanish]. Rev Peru Med Exp Salud Publica 35: 610-619.

14. Izurieta RO, Macaluso M, Watts DM, Tesh RB, Guerra B, Cruz LM, Galwankar S, Vermund SH, 2011. Hunting in the rainforest and Mayaro virus infection: an emerging Alphavirus in Ecuador. $J$ Glob Infect Dis 3: 317-323.

15. Johnson BW, Kosoy O, Wang E, Delorey M, Russell B, Bowen RA, Weaver SC, 2011. Use of sindbis/eastern equine encephalitis chimeric viruses in plaque reduction neutralization tests for arboviral disease diagnostics. Clin Vaccine Immunol 18: 1486-1491.
16. Vittor AY et al., 2016. Epidemiology of emergent Madariaga encephalitis in a region with endemic Venezuelan equine encephalitis: initial host studies and human crosssectional study in Darien, Panama. PLoS Negl Trop Dis 10: e0004554.

17. Lennette EH, Lennette DA, Lennette ET, 1995. Diagnostic Procedures for Viral, Rickettsial, and Chlamydial Infections. Washington, DC: American Public Health Association.

18. Therneau T, Atkinson B, 2018. Rpart: Recursive Partitioning and Regression Trees. Available at: https://CRAN.R-project.org/ package=rpart. $R$ Package Version $4.1-13$.

19. R Core team, 2019. R: A Language and Environment for Statistical Computing. Vienna, Austria: R Foundation for Statistical Computing.

20. Aguilar PV, Estrada-Franco JG, Navarro-Lopez R, Ferro C, Haddow AD, Weaver SC, 2011. Endemic Venezuelan equine encephalitis in the Americas: hidden under the dengue umbrella. Future Virol 6: 721-740.

21. Arrigo NC, Adams AP, Weaver SC, 2010. Evolutionary patterns of eastern equine encephalitis virus in north versus south America suggest ecological differences and taxonomic revision. $J$ Virol 84: 1014-1025.

22. Quiroz E, Aguilar PV, Cisneros J, Tesh RB, Weaver SC, 2009. Venezuelan equine encephalitis in Panama: fatal endemic disease and genetic diversity of etiologic viral strains. PLOS Negl Trop Dis 3: e472.

23. Hoch AL, Peterson NE, LeDuc JW, Pinheiro FP, 1981. An outbreak of Mayaro virus disease in Belterra, Brazil. III. entomological and ecological studies. Am J Trop Med Hyg 30: 689-698.

24. Auguste AJ et al., 2015. Evolutionary and ecological characterization of Mayaro virus strains isolated during an outbreak, Venezuela, 2010. Emerg Infect Dis 21: 1742-1750.

25. Schaeffer M, Gajdusek DC, Lema AB, Eichenwald H, 1959. Epidemic jungle fevers among Okinawan colonists in the Bolivian rain forest I. Epidemiology. Am J Trop Med Hyg 8: 372-396.

26. LeDuc JW, Pinheiro FP, Travassos da Rosa AP, 1981. An outbreak of Mayaro virus disease in Belterra, Brazil. II. Epidemiology. Am J Trop Med Hyg 30: 682-688.

27. Black FL et al., 1974. Evidence for persistence of infectious agents in isolated human populations. Am J Epidemiol 100: 230-250.

28. Talarmin A et al., 1998. Mayaro virus fever in French Guiana: isolation, identification, and seroprevalence. Am J Trop Med Hyg 59: 452-456.

29. Hotez PJ, Murray KO, 2017. Dengue, West Nile virus, Chikungunya, Zika-and now Mayaro? PLoS Negl Trop Dis 11: e0005462.

30. Calisher CH, Gutierrez E, Maness KS, Lord RD, 1974. Isolation of Mayaro virus from a migrating bird captured in Louisiana in 1967. Bull Pan Am Health Organ 8: 243-248.

31. de Thoisy B, Gardon J, Salas RA, Morvan J, Kazanji M, 2003. Mayaro virus in wild mammals, French Guiana. Emerg Infect Dis 9: 1326-1329.

32. Batista PM, Andreotti R, Chiang JO, Ferreira MS, Vasconcelos PF, 2012. Seroepidemiological monitoring in sentinel animals and vectors as part of arbovirus surveillance in the state of Mato Grosso do Sul, Brazil. Rev Soc Bras Med Trop 45: 168-173.

33. Travassos da Rosa JFS, Travassos da Rosa APA, Vasconcelos PFC, Pinheiro FP, Rodrigues SG, Travassos da Rosa ES, Dias L, Cruz A, 1988. Arboviruses isolated in the Evandro Chagas Institute, including some described for the first time in the Brazilian Amazon region, their known host, and their pathology for man. Travassos da Rosa APA, Vasconcelos PFC, Travassos da Rosa JFS, eds. An Overview of Arbovirology in Brazil and Neighbouring Countries. Belem, Brazil: Instituto Evandro Chagas, 19-31.

34. Ramírez JE, Yanoviak SP, Lounibos LP, Weaver SC, 2007. Distribución vertical de Haemagogus janthinomys (dyar) (Diptera: Culicidae) en bosques de la Amazonla Peruana. Rev Peru Med Exp Salud publica 24: 40-45.

35. Long KC, Ziegler SA, Thangamani S, Hausser NL, Kochel TJ, Higgs S, Tesh RB, 2011. Experimental transmission of 
Mayaro virus by Aedes aegypti. Am J Trop Med Hyg 85: 750-757.

36. Turell MJ, Sardelis MR, Jones JW, Watts DM, Fernandez R, Carbajal F, Pecor JE, Klein TA, 2008. Seasonal distribution, biology, and human attraction patterns of mosquitoes (Diptera: Culicidae) in a rural village and adjacent forested site near Iquitos, Peru. J Med Entomol 45: 1165-1172.

37. Brustolin M, Pujhari S, Henderson CA, Rasgon JL, 2018. Anopheles mosquitoes may drive invasion and transmission of Mayaro virus across geographically diverse regions. PLoS Negl Trop Dis 12: e0006895.

38. Cardozo F et al., 2018. Alphaviruses: serological evidence of human infection in Paraguay (2012-2013). Vector-Borne Zoonotic Dis 18:5, 266-272.

39. Martins KA et al., 2019. Neutralizing antibodies from convalescent Chikungunya virus patients can cross-neutralize Mayaro and Una viruses. Am J Trop Med Hyg 100: 1541-1544. 